\title{
PHƯƠNG PHÁP TÌM KIẾM CÁC TRỊ ĐO THÔ TRONG QUÁ TRÌNH TÍNH TOÁN BÌNH SAI TRUY HỔI VỚI PHÉP BIẾN ĐỐI XOAY ĐỐI VỚI MẠNG LƯớI Độ CAO HẠNG I, II QUỐC GIA
}

\author{
ThS. NGUYẼ̃N TH! THANH HƯO'NG \\ Viện Khoa học Đo đạc và Bản đồ
}

\section{Tóm tắt:}

Bài báo này nghiên cứu phương pháp tìm kiếm các trị đo thô dựa trên nguyên tắc mođun cực tiểu trong quy trình của phương pháp bình sai truy hồi với phép biến đổi xoay và thử nghiệm tìm kiếm các trị đo thô trong mạng lưới độ cao hạng I, II quốc gia.

\section{1. Đặt vấn đề}

Để áp dụng những phương pháp bình phương nhỏ nhất trong việc bình sai mạng lưới độ cao quốc gia, dựa trên Định lý Gauss - Markov, các trị đo của mạng lưới độ cao bao gồm các chênh cao đo hoặc các hiệu của các đại lượng địa thế năng phải được loại bỏ các sai số hệ thống và các sai số thô. Các sai số hệ thống được loại bỏ nhờ hiệu chỉnh các chênh cao đo bởi các số cải chính do biến dạng nhiệt của chiều dài trung bình 1 mét của cặp mia, các chênh cao đo hoặc các hiệu của các đại lượng địa thế năng bởi các số cải chính chuyển từ trường trọng lực thực của Quả đất về trọng trường chuẩn của elipsoid quy chiếu và từ hệ triều trung bình về hệ triều 0 .

Đối với mạng lưới độ cao quốc gia, các sai số thô xuất hiện trong các chênh cao đo hoặc các hiệu của các đại lượng địa thế năng chủ yếu do sai lầm của các kỹ thuật viên khi đưa các trị đo này vào CSDL Trị đo. Theo tài liệu (Tukey I. W. (1962)), trong các dữ liệu thực tế thường chứa khoảng $10 \%$ các dữ liệu thô. Việc kiểm tra sự có mặt và tìm kiếm các trị đo thô là tiêu chuẩn bắt buộc của các thuật toán bình sai các mạng lưới trắc địa. Thuật toán T với phép biến đổi xoay trung bình hoàn toàn có khả năng kiểm tra sự có mặt của các trị đo thô trong quá trình đưa các trị đo vào tính toán bình sai truy hồi. Vấn đề đặt ra sẽ được giải quyết trong bài báo này.

Trên thế giới việc tìm kiếm các chênh cao thô trong các mạng lưới thuỷ chuẩn rất được quan tâm. Chúng ta sẽ xem xét một phương pháp được trình bày trong tài liệu (Smoliarov I. A. (2013)).

Như đã chứng minh trong tài liệu (Hà Minh Hoà (2013)), vectơ số cải chính V được biểu diễn dưới dạng:

$$
V=-G \cdot \Delta,
$$

ở đây $\Delta$ - vectơ sai số thực của các trị đo, $G=E-A R^{-1} A^{T} P$ - ma trận có tính chất luỹ đẳng có hạng bằng số lượng các trị đo dư $\mathrm{r}, A$ - ma trận hệ số của các phương trình số cải chính, $\quad R=A^{T} P A \quad$ - ma trận chuẩn, $\mathrm{P}$ - ma trận trọng số, $\mathrm{E}$ - ma trận đơn vị bậc $\mathrm{n}$, còn $\mathrm{n}$ - tổng số các trị đo trong mạng lưới trắc địa.

Trên cơ sở công thức (1), trong tài liệu (Smoliarov I. A. (2013)) đã biểu diễn vectơ số cải chính $V_{i}$ của trị đo thứ i dưới dạng:

$$
V_{i}=-\sum_{j=1}^{n} g_{i j} \cdot \Delta_{j},
$$

ở đây $g_{i j}$ - thành phần ở hàng I cột $\mathrm{j}$ của ma trận $G, \Delta_{j}$ - sai số thực của trị đo thứ j, thêm vào đo giá trị $\Delta_{j}$ được xác định theo công thức:

Người phản biện: TS. Nguyễn Đình Thành 


$$
\Delta_{j}=-\frac{V_{j}}{g_{j j}} .
$$

Từ (2) chúng ta có biểu thức:

$$
p_{i} \cdot V_{i}=-\sum_{j=1}^{n} p_{i} \cdot g_{i j} \cdot \Delta_{j},
$$

ở đây $g_{i j}$ - thành phần đường chéo thứ j của ma trận $G$.

Nếu trị đo thứ i, ngoài sai số thực $\Delta_{i}$ còn chứa sai số thô, thì giá trị $p_{i} \cdot g_{i i} \cdot \dot{\Delta}_{i}$ sẽ nằm ở hàng thứ $\mathrm{i}$. Khi đó từ (4) chúng ta thấy rằng đại lượng $\left|p_{i} \cdot V_{i}\right|$ sẽ có giá trị lớn nhất. Chúng ta sẽ tìm giá trị gần đúng của sai số thô theo công thức (3) đối với hàng thứ i và hiệu chỉnh trị đo thứ i sau khi loại bỏ sai số thô $\Delta_{i}$. Bình sai lại mạng lưới và kiểm tra sai số thô theo điều kiện $\Delta_{i} \leq 2,5 \cdot \mu_{0} \cdot \sqrt{1 / p_{i}}$, ở đây $\mu_{0}$ - sai số trung phương trị đo được xác định một cách tiên nghiệm trước khi bình sai. Nếu điều kiện trên thoả mãn, thì quá trình tìm kiếm trị đo thô kết thúc.

Trong trường hợp ngược lại, từ kết quả bình sai lần thứ hai chúng ta xác định được giá trị cực đại của đại lượng $\left|p_{k} \cdot V_{k}\right|$ trên hàng thứ $k$. Tiếp theo, chúng ta lập hệ phương trình:

$$
\begin{aligned}
& g_{i i} \Delta_{i}+g_{i k} \cdot \Delta_{k}=-V_{i}, \\
& g_{k i} \cdot \Delta_{i}+g_{k k \cdot} \cdot \Delta_{k}=-V_{k},
\end{aligned}
$$

ở đây các giá trị $V_{i}, V_{k}$ được xác định từ lần bình sai thứ nhất.

Giải hệ phương trình (5), chúng ta tìm được các đại lượng $\Delta_{i}, \Delta_{k}$ và hiệu chỉnh các trị đo thứ i và thứ $\mathrm{j}$. Sau đó bình sai lại mạng lưới và kiểm tra điều kiện $\Delta_{k} \leq 2,5 \cdot \mu_{0} \cdot \sqrt{1 / p_{k}}$. Nếu điều kiện trên thoả mãn, thì quá trình tìm kiếm trị đo thô kết thúc. Trong trường hợp ngược lại, từ kết quả bình sai lần thứ ba chúng ta xác định được giá trị cực đại của đại lượng $\left|p_{h} \cdot V_{h}\right|$ trên hàng thứ h. Tiếp theo, chúng ta lập hệ phương trình:

$$
\begin{aligned}
& g_{i i} \cdot \Delta_{i}+g_{i k} \cdot \Delta_{k}+g_{i h} \cdot \Delta_{h}=-V_{i}, \\
& g_{k i} \cdot \Delta_{i}+g_{k k} \cdot \Delta_{k}+g_{k h} \cdot \Delta_{h}=-V_{k}, \\
& g_{h i} \cdot \Delta_{i}+g_{h k} \cdot \Delta_{k}+g_{h h} \cdot \Delta_{h}=-V_{h},
\end{aligned}
$$

ở đây các giá trị $V_{i}, V_{k}, V_{h}$ được xác định từ kết quả bình sai lần thứ nhất.

Giải hệ phương trình (6), chúng ta tìm được các đại lượng $\Delta_{i}, \Delta_{k}, \Delta_{h}$ và hiệu chỉnh các trị đo thứ i, thứ j và thứ $h$. Sau đó bình sai lại mạng lưới và kiểm tra điều kiện $\Delta_{h} \leq 2,5 . \mu_{0} \sqrt{1 / p_{h}}$. Quá trình cứ tiếp tục cho đến khi không phát hiện được các trị đo thô.

Chúng ta có thể nhận xét rằng phương pháp tìm kiếm trị đo thô nêu trên không hiệu quả vì các nguyên nhân sau:

- Quá trình phát hiện sự có mặt của các trị đo thô trong mạng lưới chỉ có thể xác định được sau khi bình sai xong mạng lưới trên cơ sở so sánh sai số trung phương đơn vị trọng số sau bình sai $\tilde{y j}^{2}$ với giá trị $\mu_{0}$. Khi

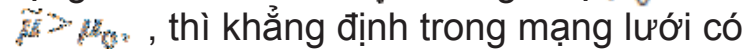
các trị đo thô. Trong khi đó, phương pháp bình sai truy hồi cho phép xác định sự có mặt của các trị đo thô ngay trong quá trình tính toán bình sai, chứ không phải đợi đến khi kết thúc quá trình bình sai.

- Ma trận $\mathrm{G}$ bậc $\mathrm{n} \times \mathrm{n}$ là ma trận đầy, nên việc xác định ma trận $G$ làm tăng thêm bộ nhớ của máy tính, đặc biệt không hiệu quả đối với mạng lưới trắc địa lớn.

Tuy nhiên, để giảm khối lượng và thời gian tính toán, trong tài liệu (Smoliarov I. A. (2013)) đã sử dụng các đường thuỷ chuẩn nối các điểm nút của mạng lưới thuỷ chuẩn để tìm kiếm các trị đo thô.

Trong Trắc địa đã có đề xuất sử dụng các điều kiện của cả phương pháp bình phương nhỏ nhất lẫn phương pháp modun cực tiểu (phương pháp đánh giá $M$ ) để bình sai ổn định mạng lưới trắc địa có chứa các trị đo thô trên cơ sở cục bộ hoá các trị đo thô nhằm nhận được các đánh giá các tham số 
ẩn không xê dịch, ví dụ xem (larmolenko A.X. (1992)). Như đã chỉ ra trong (Hà Minh Hoà (2013)), phương pháp bình sai ổn định mạng lưới trắc địa được nghiên cứu ở trên có nhược điểm cơ bản: phương pháp này không tính đến việc cục bộ hoá các trị đo thô theo phương pháp đánh giá $\mathrm{M}$ để nhận được vectơ $\delta X$ không xê dịch sẽ làm mất đi các thông tin quý giá về các trị đo (tương ứng với các trị đo thô), đặc biệt khi các trị đo thô xuất hiện do sai lầm khi đưa dữ liệu vào máy tính điện tử, liên quan đến việc giảm số lượng các trị đo dư trong mạng lưới trắc địa. Ngoài ra việc còn tồn tại các trị đo thô trong CSDL trắc địa sẽ gây khó khăn trong việc sử dụng CSDL này để phát triển mạng lưới trắc địa. Điều này giải thích sự không phổ biến của việc bình sai ổn định mạng lưới trắc địa trong thực tế tính toán bình sai các mạng lưới trắc đại hiện nay.

Trong bài báo này chúng ta sẽ nghiên cứu phương pháp tìm kiếm các trị đo thô hiệu quả hơn dựa trên nguyên tắc mođun cực tiểu được đề xuất trong tài liệu (Hà Minh Hoà (2013)).

\section{Giải quyết vấn đề}

Như đã trình bày trong tài liệu (Hà Minh Hoà (1996)); (Hà Minh Hoà (2013)); (Hà Minh Hoà (2014)), phân bố Laplace phù hợp khi phương sai của các trị đo thay đổi từ 0 đến vô cực. Như vậy, các sai số thô của các trị đo sẽ tuân theo phân bố Leplace. Khi đó để tìm kiếm các trị đo thô (các trị đo có chứa các sai số thô) là điều kiện mođun cực tiểu ở dạng dưới đây:

$$
\sum_{i=1}^{n}\left|\bar{v}_{i}\right|=\min ,
$$

ở đây $\bar{v}_{i}=p_{i}^{1 / 2} \cdot v_{i}$, còn $p_{i}$ là trọng số đo của trị đo $y_{i}$.

Để triển khai điều kiện (7), khi tính đến sự phổ biến của phương pháp bình phương nhỏ nhất truyền thống trong việc xử lý toán học các kết quả đo đạc, Fletcher R., Grand J.A., Heblen M.D. trong (Fletcher R., Grand J.A, Hebden M.D. (1971)) đã đề xuất phương pháp bình phương nhỏ nhất lặp và còn được gọi là phương pháp Fletcher Grand - Hebden. Khi đó điều kiện (7) được biểu diễn lại như sau:

$$
\begin{aligned}
\sum_{i=1}^{n}\left|\bar{v}_{i}\right| & =\sum_{i=1}^{n} \bar{p}_{i} \cdot \bar{v}_{i}^{2}=\min , \\
\text { ở đây } \quad \bar{p}_{i} & =\frac{1}{\left|\bar{v}_{i}\right|} .
\end{aligned}
$$

Sự hội tụ của quá trình giải lặp theo điều kiện (2) đã được chứng minh trong tài liệu (Mudrov V.I., Kusko V.L. (1976)). Trong các tài liệu (Hà Minh Hoà (1996); Hà Minh Hòa (2013); Hà Minh Hòa (2014)) đã chứng minh được rằng sau khi quá trình bình sai lặp hội tụ với mức 0,0001 , số cải chính của các trị đo không chứa các sai số thô sẽ tiến tới 0 , còn các số cải chính của các trị đo thô sẽ tiến tới các giá trị của sai số thô. Tuy nhiên, điều này chỉ đạt được đối với các mạng lưới trắc địa dầy đặc có nhiều các trị đo dư, trừ mạng lưới đường chuyền.

Phương pháp bình sai truy hồi với phép biến đổi xoay (xem các tài liệu Hà Minh Hòa (2013); Hà Minh Hòa (2014)) cho phép phát hiện sự có mặt của các trị đo thô ngay trong quá trình tính toán bình sai truy hồi. Các ưu điểm cơ bản của phương pháp bình sai truy hồi với phép biến đổi xoay (chúng ta gọi là thuật toán T) là khả năng sử dụng kỹ thuật ma trận thưa để lưu giữ ma trận biến đổi và giảm thiểu sự tích lũy của các sai số làm tròn trong quá trình bình sai mạng lưới trắc địa lớn. Do đó, việc kết hợp phương pháp bình sai truy hồi với phép biến đổi xoay với phương pháp bình phương nhỏ nhất lặp cho phép vừa phát hiện vừa tiến hành tìm kiếm sự có mặt của các trị đo thô.

Thuật toán $T$ làm việc với ma trận tam giác trên $T$ liên hệ với ma trận chuẩn $R$ theo công thức $R=T^{\top} T$. Để chuẩn bị bình sai truy hồi, chúng ta nhận ma trận ban đầu 
$T_{0}=10^{-6} \cdot E_{\mathrm{kxk}}$, vectơ số hạng tự do $Y_{0}=0$ và tổng $\Phi_{0}=[P V V]_{0}=0$, ở đây $E_{\mathrm{kxk}}-\mathrm{ma}$ trận đơn vị bậc $\mathrm{k}$, còn $\mathrm{k}$ là tổng số ẩn số cần tìm. Sau khi đưa vào tính toán bình sai truy hồi i-1 trị đo đầu tiên, chúng ta nhận được ma trận tam giác trên $T_{\mathrm{i}}$, vectơ số hạng tự do $Y_{\mathrm{i}-1}$ và tổng $\Phi_{i-1}$.

Trị đo thứ $\mathrm{i}(\mathrm{i}=1,2, \ldots, \mathrm{n}$, còn $\mathrm{n}$ - tổng số các trị đo trong mạng lưới trắc địa) là $y_{i}$ với trọng số $p_{i}$ có phương trình số cải chính dạng $v_{i}=a_{i} \cdot \delta X_{i}+l_{i}^{(0)}$, ở đây $a_{\mathrm{i}}$ - ma trận hàng chứa các hệ số của phương trình số cải chính, $l_{i}^{(0)}=\varphi_{i}\left(X^{(0)}\right)-y_{i}, \quad X^{(0)}$ - vectơ các giá trị khái lược của các tham số ẩn cần tìm, $\varphi_{i}\left(X^{(0)}\right)$ - hàm liên hệ. Khi đưa trị đo $y_{\mathrm{i}}$ vào tính toán bình sai truy hồi theo thuật toán $T$, chúng ta nhận được ngay các kết quả bình sai bao gồm ma trận tam giác trên được biến đổi $T_{\mathrm{i}}$, vectơ số hạng tự do được biến đổi $Y_{\mathrm{i}}$ và tổng $\Phi_{i}=[P V V]_{i}$ mà không phải lập và giải hệ phương trình chuẩn.

Để kiểm tra sự có mặt của các trị đo thô, chúng ta xác định vectơ $t_{\mathrm{i}}$ từ giải hệ

$$
T_{i-1}^{T} \cdot t_{i}=a_{i}^{T} .
$$

Tiếp theo xác định số hạng tự do $l_{i}=t_{i}^{T} \cdot Y_{i-1}+l_{i}^{(0)}$ và xác định trọng số đảo của nó lớn hơn $g_{i}=p_{i}^{-1}+t_{i}^{T} \cdot t_{i}$. Nếu $g_{i} \leq \frac{100}{p_{i}}$, thì trị đo $y_{\mathrm{i}}$ là trị đo dư. Khi đó, chúng ta kiểm tra sự có mặt của trị đo thô trên cơ sở so sánh số hạng tự do với hạn sai của nó $\left(l_{i}\right)_{g h}=3 \cdot m_{0} \cdot \sqrt{g_{i}}$, ở đây $m_{0}$ - sai số trung phương của các trị đo được đánh giá từ trước một cách tiên nghiệm.

Nếu $I_{i}>\left(I_{0}\right)_{g h}$, thì trong các trị đo được đưa vào tính toán bình sai truy hồi có tồn tại trị đo thô.

Để tìm kiếm trị đo thô theo phương pháp bình phương nhỏ nhất lặp, phương trình số cải chính của trị đo $y_{i}$ được đưa vào quá trình tính toán bình sai truy hồi lặp lần thứ $\mathrm{m}$ có dạng:

$$
\bar{v}_{i}^{(m)}=\bar{a}_{i} \cdot \delta X^{(m)}+\bar{l}_{i}^{(0)},
$$

ở đây ma trận hệ số $\bar{a}_{i}=p_{i}^{1 / 2} \cdot a_{i}$; số hạng tự do $\bar{l}_{i}^{(0)}=p_{i}^{1 / 2} l_{i}^{(0)}$.

Chúng ta sẽ tiến hành tìm kiếm sự có mặt của các trị đo thô nhờ bình sai lặp dưới điều kiện:

$$
\sum_{i=1}^{n} \bar{p}_{i}^{(m)} \cdot\left(\bar{v}_{i}^{(m)}\right)^{2}=\min ,
$$

Ở đây $\mathrm{m}=1,2, \ldots$ là số thứ tự lần bình sai lặp, $\bar{p}_{i}^{(m)}=\frac{1}{\left|\bar{v}_{i}^{(m-1)}\right|}$, thêm vào đó số cải chính của trị đo $(i=1,2, \ldots, n)$ được xác định theo công thức

$$
\bar{v}_{i}^{(m-1)}=p_{i}^{1 / 2} \cdot\left(\varphi_{i}\left(X^{(0)}+\delta X^{(m-1)}\right)-y_{i}\right),
$$

$X^{(0)}$ là vectơ các giá trị khái lược của các tham số ẩn (các giá trị khái lược của các độ cao chuẩn hoặc các đại lượng địa thế năng của các mốc độ cao); $p_{\mathrm{i}}$ là trọng số của trị đo $y_{i} ; \delta X^{(m-1)}$ là vectơ các số cải chính của các tham số ẩn nhận được trong quá trình bình sai lặp thứ $(\mathrm{m}-1)$ với lưu ý rằng $\delta X^{(0)}=0$; $\varphi_{i}(X)$ là hàm liên hệ giữa các tham số ẩn và trị đo $y_{\mathrm{i}}$

Quá trình bình sai lặp sẽ dừng lại khi các số cải chính của các trị đo giữa hai lần bình sai lặp chênh nhau một giá trị nhỏ bỏ qua (ở mức 0,0001$)$. Như đã chứng minh trong tài liệu ((Hà Minh Hòa (2013)), đối với các trị đo không chứa sai số thô, sau quá trình $\mathrm{m}$ lần bình sai lặp các số cải chính của chúng sẽ tiến đến số 0 . Trái lại, đối với các trị đo thô, các số cải chính sẽ tiến đến các giá trị của các sai số thô (lớn hơn 3 lần sai số trung phương của các trị đo trong mạng lưới trắc địa).

Việc tìm kiếm một cách tin cậy các trị đo 
thô trong mạng lưới trắc địa phụ thuộc vào lượng đo dư trong mang lưới đó. Trong thực tế, chúng ta có thể tìm được một trị đo thô với điều kiện trị đo thô đó có mặt trong hai phương trình điều kiện. Như vậy để tìm được một trị đo thô đòi hỏi phải có ít nhất 02 trị đo dư trong mạng lưới trắc địa. Với đặc điểm nêu trên của việc tìm kiếm trị đo thô trong mạng lưới trắc địa, chúng ta nhận thấy rằng đối với một đường độ cao bao gồm nhiều đoạn độ cao và có hai mốc cứng là hai mốc đầu và cuối của đường, chúng ta không thể tìm được đoạn độ cao chứa trị đo thô trong đường đó. Do đó, trong thực tế bình sai mạng lưới độ cao hạng I, II quốc gia, chúng ta có hai bước tính toán bình sai:

Bước 1. Tạo mạng lưới độ cao với các mốc cần xác định là các điểm nút nối các đường độ cao. Kiểm tra sự có mặt của đường độ cao có chứa các trị đo thô (các chênh cao đo thô hoặc các hiệu các đại lượng địa thế năng đo thô) được thực hiện theo thuật toán $T$ - thuận. Nếu không phát hiện được các đường độ cao có chứa các trị đo thô, chúng ta chuyển sang thực hiện bước 2 ở dưới đây. Trong trường hợp ngược lại, chúng ta tiến hành tìm kiếm chúng theo phương pháp mô đun cực tiểu với điều kiện (7). Sau khi tìm kiếm được đường độ cao có chứa các trị đo thô, chúng ta tiến hành tìm các trị đo thô trên các đoạn độ cao của đường đó từ các tài liệu đo đạc và tính toán khái lược. Sau khi đã phát hiện được các trị đo thô và sửa chữa chúng, chúng ta sẽ chuyển sang bước 2 ở dưới đây.

Bước 2. Bình sai mạng lưới độ cao quốc gia với các mốc cần xác định là toàn bộ các mốc độ cao hạng I, II quốc gia.

Như vậy, việc phát hiện và tìm kiếm các trị đo thô trong mạng lưới độ cao hạng I, II quốc gia làm cho quy trình bình sai mang lưới này phức tạp thêm. Tuy nhiên, đây là cái giá phải trả để nhận được các kết quả bình sai tin cậy nhất đáp ứng các yêu cầu của định lý Gauss - Markov.

\section{Thực nghiệm}

Để thực nghiệm phương pháp tìm kiếm các trị đo thô trong mạng lưới độ cao, chúng ta sử dụng mạng lưới độ cao ở hình dưới đây.

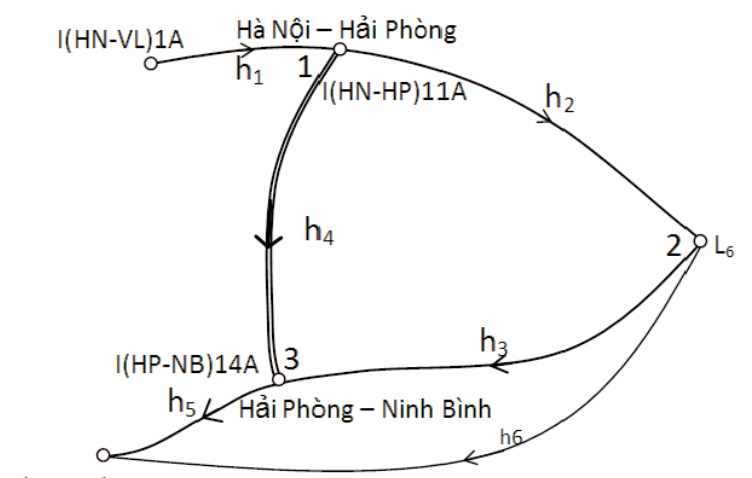

I(HN-VL) $16 \mathrm{~A}$

* Số liệu gốc

\begin{tabular}{|c|c|}
\hline Tên điểm & Độ cao \\
\hline $\mathrm{I}(\mathrm{HN}-\mathrm{VL}) 1 \mathrm{~A}$ & 5,4929 \\
\hline $\mathrm{I}(\mathrm{HN}-\mathrm{VL}) 16 \mathrm{~A}$ & 1,6205 \\
\hline
\end{tabular}

* Số liệu đo:

\begin{tabular}{|c|c|c|c|}
\hline Điểm đầu & Điểm cuối & Chênh cao & $\begin{array}{c}\text { Chiều dài } \\
\text { tuyến }\end{array}$ \\
\hline I(HN-VL)1A & I(HN-HP)11A & $-2,79584$ & 69,9 \\
\hline I(HN-HP)11A & L6 & $-2,10076$ & 55,8 \\
\hline L6 & I(HP-NB)14A & 0,21246 & 84,1 \\
\hline I(HN-HP)11A & I(HP-NB)14A & $-1,87836$ & 105,1 \\
\hline I(HP-NB)14A & I(HN-VL)16A & 0,81900 & 62,3 \\
\hline L6 & I(HN-VL)16A & 1,02000 & 70,1 \\
\hline
\end{tabular}

Giả định trị đo cuối (L6-> I(HN-VL)16A có chứa sai số thô là $1.00 \mathrm{~m}$, giá trị chênh cao lúc này là $2.02000 \mathrm{~m}$

Ta có hệ phương trình số hiệu chỉnh: 


\begin{tabular}{|c|c|c|c|c|}
\hline H1 & H2 & H3 & L & P \\
\hline 1 & 0 & 0 & 0,0000 & 0,0143 \\
\hline-1 & 1 & 0 & 0,0000 & 0,0179 \\
\hline 0 & -1 & 1 & 0,0000 & 0,0119 \\
\hline-1 & 0 & 1 & $-0,0099$ & 0,0095 \\
\hline 0 & 0 & -1 & $-0,0073$ & 0,0161 \\
\hline 0 & -1 & 0 & $-0,9958$ & 0,0143 \\
\hline
\end{tabular}

Tác giả đã viết một chương trình máy tính với thuật toán trên, sau khi chạy thực nghiệm với hệ phương trình số hiệu chỉnh trên, qua 9 lần lặp thì chương trình chỉ ra 3 trị đo có khả năng là chứa sai thô.

\begin{tabular}{|c|c|c|}
\hline Lần lặp thứ: 9 & Số hiệu chỉnh & Nhận định \\
\hline $\mathrm{V}:=1$ & 0,017199235 & Trị đo 1 có thể là trị đo thô \\
\hline $\mathrm{V}:=2$ & 0,00011479 & \\
\hline $\mathrm{V}:=3$ & 0,010054043 & Trị đo 3 có thể là trị đo thô \\
\hline $\mathrm{V}:=4$ & $7,47099 \mathrm{E}-07$ & \\
\hline $\mathrm{V}:=5$ & $1,82997 \mathrm{E}-08$ & \\
\hline $\mathrm{V}:=6$ & 0,978485975 & Trị đo 6 có thể là trị đo thô \\
\hline
\end{tabular}

Trong đó số hiệu chỉnh của trị đo thứ 6 là lớn nhất và gần với giá trị sai thô $(\mathrm{V} 6=0$, $978485975 \sim 1.000$ ).

\section{Kết luận}

Kết hợp khả năng kiểm tra sự có mặt của các trị đo thô trong quá trình tính toán bình sai truy hồi theo Thuật toán $T$ thuận với nguyên tắc mô đun cực tiểu cho phép giải quyết hiệu quả bài toán phát hiện và tìm kiếm các trị đo thô trong mạng lưới độ cao quốc gia. Trong trường hợp này, đối với mạng lưới độ cao hạng I, II quốc gia cần tạo mạng lưới các điểm nút bao gồm các đường độ cao giữa các điểm nút và việc tìm kiếm các đường có chứa các chênh cao thô được thực hiện theo phương pháp bình sai truy hồi với phép biến đổi xoay kết hợp với việc bình sai lặp theo nguyên tắc mô đun cực tiểu. $O$

\section{Tài liệu tham khảo}

[1]. Fletcher R., Grand J.A, Hebden M.D. (1971). The calculation of linear least apporoximations. - Computer journal, 1971,v.14, No3, pp. 277-279.

[2]. Hà Minh Hoà (1996). Sử dụng hàm Huber P.J. để tìm kiếm trị đo thô trong bình sai truy hồi. Tạp chí Trắc địa và Bản đồ. N2,1996,trg. 22 - 24. Hội Trắc địa - Bản đồ - Viễn thám Việt Nam.

[3]. Hà Minh Hòa (2013). Phương pháp bình sai truy hồi với phép biến đổi xoay. Sách chuyên khảo. NXB Khoa học và Kỹ thuật, 287 trg. Hà Nội.

[4]. Hà Minh Hòa (2014). Phương pháp xử lý toán học các mạng lưới trắc địa quốc gia. Nhà Xuất bản Khoa học và Kỹ thuật, 244 trg. Hà Nội - 2014.

[5]. Iarmolenko A.X. (1992). Phương pháp bình sai ổn định các mạng lưới trắc địa mặt bằng. Izv. Vuzov. Geodezia i Aerophotoxemka. № 2, 1992, trg. 48-63. (Tiếng Nga).

[6]. Mudrov V.I., Kusko V.L. (1976). Các phương pháp hiệu chỉnh đo đạc. M. Xov. Radio, 1976, 192 trg. (Tiếng Nga).

[7]. Smoliarov I.A. (2013). Vấn đề phát hiện và nhận diện các sai số đo đạc thô theo các kết quả bình sai các mạng lưới thủy chuẩn bằng phương pháp gián tiếp. Trong quyển sách: Trắc địa Vật lý. Tuyển tập khoa học - kỹ thuật TXNHIIGAiK, Matxcơva, Thế giới khoa học, 2013, 288 trg.

[8]. Tukey I.W. (1962). The future of data analysis. Ann. Math. Statist. 1962,vol. 33, pp. 1-67.0

(Xem tiếp trang 52) 